\section{Medicinal Plants of South Africa}

by Ben-Erik Van Wyk, Bosch van Oudtshoorn and Nigel Gericke: Briza Publications, Pretoria, South Africa, 2nd edn., 2009, pp 336, ISBN 978-1-875093-37-3.

Price: ZAR 329.00 (appr. GBP 29.50, EUR 33.20)

The first edition of this book, published in 2002, deservedly won the 2003 Noma Award for Publishing in Africa, where the jury's report described it as "A beautifully produced and illustrated book presented in a clear, concise, and well-laid out manner; it is accessible, but at the same time scholarly and authoritative". Now with a small change of authorship, Fanie van Heerden being replaced by Nigel Gericke, the second edition has appeared with a new cover featuring the Asclepiad Hoodia pillansii (noted for its reputed effect as a 'slimming pill' or hunger-suppressant). The plant is correctly assigned to the family Apocynaceae, following the latest systematic placement of APGIII, an example of how the new edition has been carefully revised and brought up-to-date.

The main text of the book consists of a page of well-referenced botanical, medicinal and pharmacological information, accompanied by a distribution map and chemical formula diagrams, faced by a page of full-colour photographs. These have been well chosen to show the plant's habit, floral details, storage organs (seeds, roots, etc.) and in some cases views of the plant or its bark in the form seen when harvested. In a few cases product packaging is shown. This makes the work far more useful as a reference source than most Southern African floras, and will help to encourage sales to a wider range of libraries than would be the case for a strictly taxonomic treatment.

Some space has been wasted in the bibliographic footnotes due to the fact that frequently cited sources are given in full on each occasion when they are mentioned; and the fact that the text is constrained to one page per species forces the authors to adopt a rather telegraphic style when dealing with species with a multitude of uses.

For me, the most interesting part of the book was the introductory material, with chapters describing the importance of medicinal plants (including some not native to South Africa), cultural aspects of healing, methods of collection and storage and dosage forms. It is clear that there is a lot to be learned from the usages of native South African plants which may be relevant to other, related species occurring elsewhere. There is also considerable export potential, with cer- tain species such as Harpagophytum procumbens (Devil's Claw) already being used in considerable quantities worldwide. This brings me to the one important aspect that the book largely overlooks, namely the conservation status of rare wild plants in South Africa; finding a balance between exploitation and conservation is the key to a sustainable industry, and although some entries mention that the products are expensive due to the rarity of the source material, there is no further information on conservation measures such as legal restrictions on collecting, though in a few cases such as Warburgia salutaris (Pepperbark Tree) the need for cultivation is mentioned.

The book contains the obligatory warning that it "is intended as a scientific overview and not as a medicinal handbook for self-treatment". Indeed, as only 150 species are selected this is by no means a comprehensive account of South African medicinal plants, given that the flora is one of the richest in Africa with over 30,000 species. But it can be recommended to medical and botanical readers both as a convenient source of summary information and as an effective means of locating relevant literature.

John Edmondson 